\title{
Minimum Dissection of a Rectilinear Polygon with Arbitrary Holes into Rectangles
}

\author{
Valeriu Soltan and Alexei Gorpinevich \\ Institute of Mathematics, Moldavian Academy of Sciences, Grosul str 5, \\ Kishinev 277028, Republica Moldova
}

\begin{abstract}
In this paper, the problem of dissecting a plane rectilinear polygon with arbitrary (possibly, degenerate) holes into a minimum number of rectangles is shown to be solvable in $O\left(n^{3 / 2} \log n\right)$ time. This fact disproves a famous assertion about the NP-hardness of the minimum rectangular dissection problem for rectilinear polygons with point holes.
\end{abstract}

\section{Introduction}

The following decomposition problem is well studied in computational geometry (see, for instance, [1], [3], [4], and [6]-[9]): Given a rectilinear polygon with rectilinear holes, dissect its interior into a minimum number of nonoverlapping rectangles. In the case of nondegenerate holes this problem can be solved in $O\left(n^{5 / 2}\right)$ time, where $n$ is the number of vertices of the polygon (see [1], [4], and [6]). Lipski [7], [8] and Imai and Asano [3] gave $O\left(n^{3 / 2} \log n\right)$ algorithms for this minimum dissection problem. Both algorithms make use of the bipartite intersection graph of a set of vertical and horizontal line segments.

If the holes of a rectilinear polygon can degenerate into points, the minimum dissection problem is asserted to be NP-hard [5]. Nevertheless, we prove below the solvability of this problem in $O\left(n^{3 / 2} \log n\right)$ time in the case of arbitrary (possibly, degenerate) holes. In this way, we adapt the respective techniques developed in $[3],[4]$, and [6]-[8], which breaks down in the case of degenerate holes.

\section{Description of Rectilinear Polygons}

Let $P$ be a (possibly, multiply connected) bounded polygonal region in the coordinate plane $E$. The topological boundary bd $P$ of $P$ is assumed to be the 
union of a finite number of simple rectilinear contours. A polygonal contour is called rectilinear provided all of its line segments are either horizontal or vertical. We assume that any two of these contours will be situated either one inside the other or mutually noninclusive, that they will have common vertices but no common line segments, and that they are not interlaced.

As usual, a point $x \in E$ is called interior for $P$ provided it is contained inside an odd number of simple contours determining bd $P$. All interior points of $P$ form the topological interior int $P$.

Inside $P$ a finite family of closed line segments $s_{1}, \ldots, s_{u}$ (either horizontal or vertical), and a finite family of isolated points $v_{1}, \ldots, v_{\varepsilon}$, may be situated such that the following conditions are fulfilled:

(1) $v_{1}, \ldots, v_{t}$ belong to int $P \backslash\left(s_{1} \cup \cdots \cup s_{u}\right)$;

(2) the interior of each segment $s_{i}$ is contained in int $P$;

(3) if segments $s_{i}, s_{j}$ have a common point, then it is a vertex for both $s_{i}, s_{j}$.

The point-set union of these points and segments is called the ornament of $P$, and denoted by Or $P$.

In order to consider the degenerate holes, we introduce a nonstandard topology in the plane $E$. Since the description of the respective constructions is sometimes rather formal, we use some illustrative examples. The first of them is shown in Fig. 1.

Example. For rectilinear polygon $B$, represented in Fig. 1, bd $B$ is the union of four rectilinear contours $[1,3,10,11,24,23,32,30,1],[4,5,13,12,4]$, $[9,10,23,22,9]$, and $[16,17,26,25,16]$. We have

$$
\begin{aligned}
\text { Or } B= & \{21\} \cup\{29\} \cup[2,7] \cup[6,7] \cup[7,8] \cup[7,14] \\
& \cup[15,20] \cup[18,19] \cup[18,27] \cup[19,28] \cup[25,31] \cup[27,28]
\end{aligned}
$$

Since the segments of contour $[18,19,28,27,18]$ are defined to be in Or $P$, the interior of rectangle $[18,19,28,27]$ belongs to int $B$.

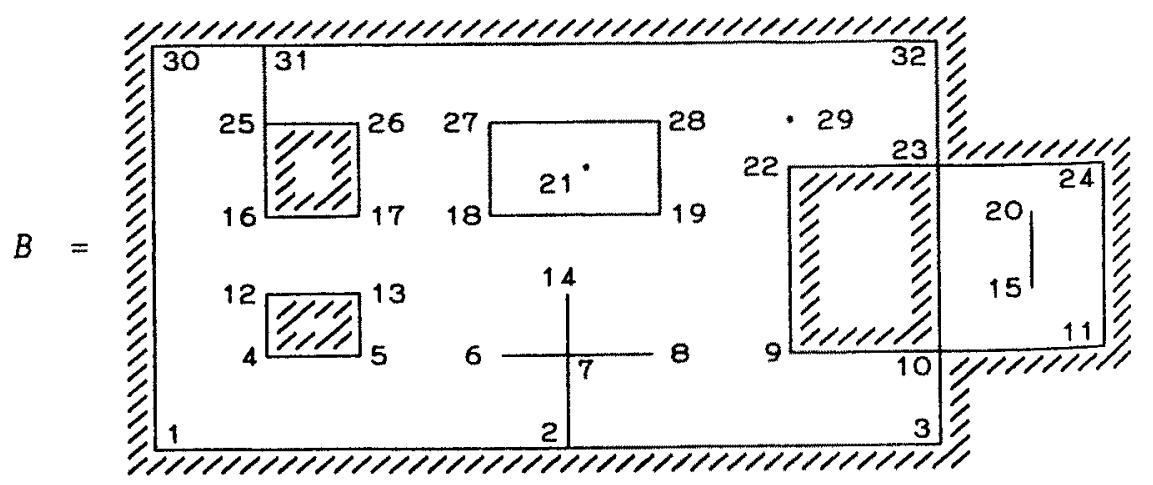

Fig. 1. A rectilinear polygon in the plane. 
Definition 1. The sets

$$
\text { Bd } P:=\text { Or } P \cup \text { bd } P, \quad \text { Int } P:=P \backslash \text { Bd } P
$$

are called, respectively, the formal boundary and the formal interior of $P$. Any bounded connected component of $E \backslash$ Int $P$ is named a formal hole of $P$, and the unbounded connected component of $E \backslash$ Int $P$ is called the formal exterior of $P$. We write Ext $P$ for the formal exterior of $P$.

From the definitions above, it follows that Bd $P$, Ext $P$, and any formal hole of $P$ are closed sets, while Int $P$ is open. As usual, a topological hole of $P$ is defined to be a bounded connected component of $E \backslash P$. Observe that topological holes are independent of the choice of Or $P$.

Example. Polygon $B$ in Fig. 1 has five formal holes: points 21 and 29 , segment $[15,20]$, rectilinear contour $[18,19,28,27,18]$, and rectangle $[4,5,13,12]$. Rectangle $[16,17,26,25]$ is not a formal hole of $B$, because it is connected with the exterior of $B$ by segment $[25,31] . B$ has three topological holes, namely, the interiors of rectangles $[4,5,13,12],[9,10,23,22]$, and $[16,17,26,25]$.

Now we are able to define the dissection of $P$ into rectangles.

Definition 2. A rectilinear polygon $P$ is said to be dissected into rectangles $R_{1}, \ldots, R_{q}$ if and only if

$$
\bigcup_{i=1}^{q} \text { int } R_{i} \subset \operatorname{Int} P \subset \bigcup_{i=1}^{q} R_{i}, \quad \text { int } r_{i} \cap \text { int } R_{j}=\varnothing \quad \text { for } \quad i \neq j
$$

In other words, nonoverlapping rectangles $R_{1}, \ldots, R_{q}$ dissect $P$ if their union coincides with $P$ and the formal boundary $\mathrm{Bd} P$ belongs to the union of $R_{1}, \ldots, R_{q}$ boundaries.

Example. A dissection into rectangles of rectilinear polygon $B$ from Fig. 1 is shown in Fig. 2.

We continue the description of rectilinear polygons.

Definition 3. A point $w$ is called a vertex of $P$ in any one of the following cases:

(1) $w$ is a vertex of a contour forming bd $P$;

(2) $w$ is a vertex of a segment $s_{i}$ forming Or $P$;

(3) $w$ is one of the isolated points $v_{i}$ in Or $P$.

The set of all vertices of $P$ is denoted by $V(P)$. 


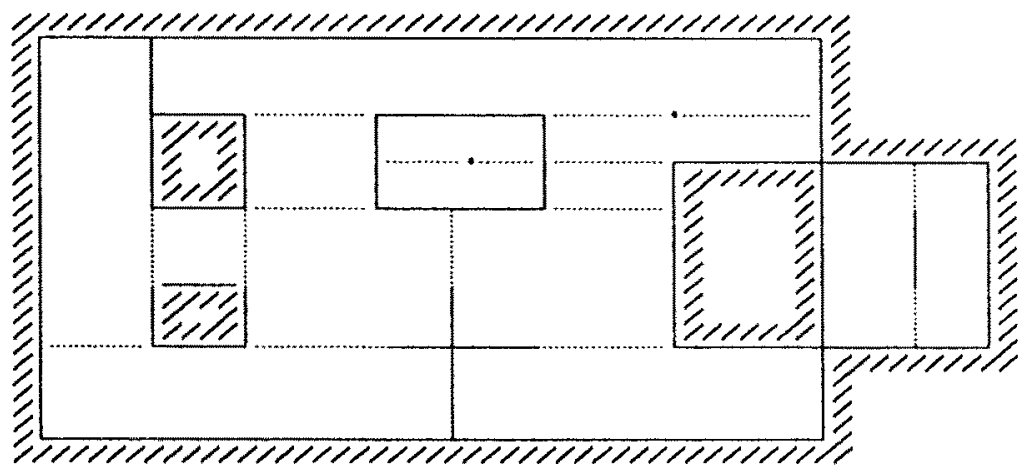

Fig. 2. A dissection of a rectilinear polygon into rectangles.

Definition 4. A closed line segment $[x, z], x \neq z$, is called an elementary segment of $\mathrm{Bd} P$ if $[x, z] \subset \mathrm{Bd} P$ and $[x, z] \cap V(P)=\{x, z\}$. The set of all elementary segments of $\mathrm{Bd} P$ is denoted by $S(P)$.

Example. Polygon $B$ in Fig. 1 has 32 vertices and 32 elementary segments. Note that $[1,3]$ and $[30,32]$ are not elementary segments of $B d B$, because they contain, respectively, vertices 2 and 31 in their interiors.

From the definitions, it follows that a common point of elementary segments $t_{i}, t_{j} \in S(P)$ is a vertex for both segments.

Observation 1. A rectilinear polygon $P$ is considered to be determined if the sets int $P, \mathrm{Bd} P$, and $V(P)$ are known. It is easily seen that $\mathrm{Bd} P$ and $V(P)$ uniquely determine $S(P)$.

\section{Measure of Local Nonconvexity}

Any vertex $v$ (if it is not an isolated point in $\mathrm{Bd} P$ ) is the apex of at least one nonempty inner angle of $P$. Every inner angle of $P$ with the apex $v$ is bounded by two elementary segments of the form $[u, v],[v, w]$, and contains no other inner angle with the apex $v$. We allow the sides of an inner angle to coincide (in this case, the size of the angle equals $2 \pi$ ). Obviously, any inner angle of a rectilinear polygon is of size $\pi k / 2, k=1,2,3,4$. Inner angles of size $3 \pi / 2$ or $2 \pi$ are called concave.

Definition 5. A vertex $v$ of $P$ is called a point of local nonconvexity of $P$ provided it is either an isolated point in $\mathrm{Bd} P$ or the apex of a concave inner angle of $P$; otherwise, $v$ is called a point of local convexity of $P$.

Example. In Fig. 1, vertex 7 is the apex of four inner angles; each of the vertices $2,10,18,19,23,27,28$, and 31 is the apex of two inner angles; each of the vertices 
$6,8,14,15$, and 20 is the apex of an inner angle of size $2 \pi$. Vertices $4-6,8,9$, $12-22$, and $26-29$ are points of local nonconvexity for $B$.

Definition 6. The measure $m(v)$ (of local nonconvexity) of a rectilinear polygon $P$ at a vertex is defined as follows:

$m(v)=0 \quad$ if $v$ is a point of local convexity of $P$;

$m(v)=1$ if $v$ is a point of local nonconvexity but not an isolated point in Bd $P$;

$m(v)=2$ if $v$ is an isolated point in $\mathrm{Bd} P$.

Let

$$
m(P):=\sum m(v),
$$

where the sum is taken over the set $V(P)$ of all vertices of $P$. The number $m(P)$ is called the measure (of local nonconvexity) of $P$.

Example. In Fig. 1, the measure of $B$ at each of the vertices 21 , and 29 equals two, at each of the vertices $4-6,8,9,12-20,22$, and $26-28$ is equal to one, and at any other vertex it equals zero. Thus $m(B)=22$.

We now state two simple lemmas that will be of use later.

Lemma 1. Any formal hole of a rectilinear polygon $P$ contains at least one vertex of local nonconvexity of $P$.

Proof. Let $H$ by any formal hole of $P$. It is easily seen that any vertex $v$ of $H$ having the maximum sum $X+Y$ of its Cartesian coordinates $(X, Y)$ is a vertex of the local nonconvexity of $P$.

Lemma 2. A rectilinear polygone $P$ has no point of local nonconvexity if and only if each component of $\mathrm{Int} P$ is an open rectangle; i.e., if $\mathrm{Bd} P$ divides $P$ into rectangles.

Proof. A rectilinear polygone $P$ has no point of local nonconvexity if and only if $\mathrm{Bd} P$ contains no isolated point and each inner angle of $P$ is of size $\pi$ or $\pi / 2$. Now, the assertion of Lemma 2 readily follows.

\section{Effective Chords}

Further we shall see that the following line segments are of significant importance in any dissection of a rectilinear polygon into a minimum number of rectangles. 
Definition 7. A line segment $[v, w]$ is called an effective chord of $P$ if the following conditions are fulfilled:

(1) $[v, w]$ is either horizontal or vertical;

(2) the open interval $] v, w$ [ with the exception of a finite (possibly, empty) set of points is contained in Int $P$;

(3) $v, w$ are vertices of local nonconvexity of $P$ and each of them is either an isolated point in $\mathrm{Bd} P$ or a vertex of an elementary segment of $\mathrm{Bd} P$ collinear to $[v, w]$

(4) if a point $x \in \mathrm{Bd} P$ belongs to $] v, w[$, then $x$ is a vertex of a unique elementary segment of $\mathrm{Bd} P$ orthogonal to $[v, w]$.

Example. Polygon $C$ (see Fig. 3) with the ornament

$$
\text { Or } C=[1,2] \cup[3,4] \cup[7,8] \cup[12,13] \cup\{5\} \cup\{10\} \cup\{11\}
$$

has the following effective chords: $[2,12],[4,5],[5,10],[6,7],[9,10]$, and $[10,11]$.

From Definition 7, it follows:

Observation 2. Any two different effective chords of $P$ have at most one common point. Any proper part of an effective chord cannot be an effective chord.

Definition 8. A family of effective chords of a rectilinear polygon $P$ is called admissible if any two mutually orthogonal chords of this family have no common point. The maximum number of effective chords forming an admissible family for $P$ is named the effective number of $P$ and is denoted by $e(P)$.

Example. Polygon $C$ in Fig. 3 has three maximal admissible families of effective chords: $\{[2,12],[5,10]\},\{[2,12],[4,5],[9,10],[10,11]\}$, and $\{[4,5],[6,7]$,

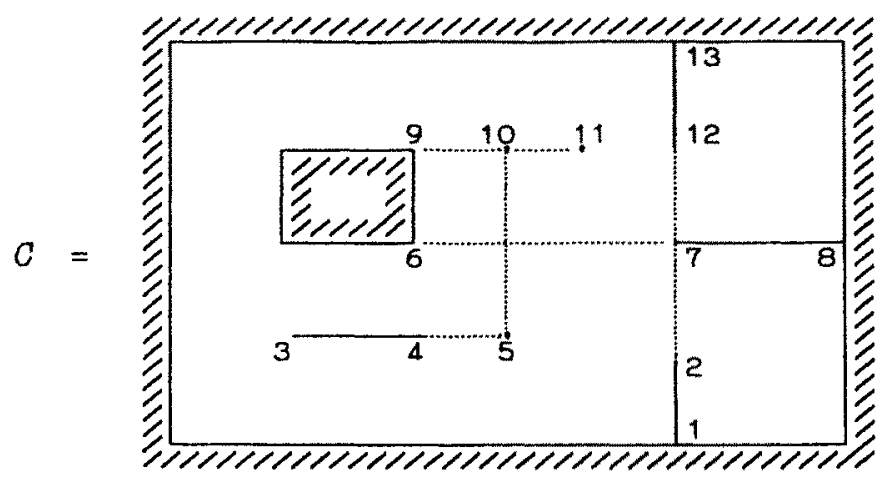

Fig. 3. Effective chords of a rectilinear polygon. 
$[9,10],[10,11]\}$. Thus $e(C)=4$, and a maximal admissible family need not be a maximum admissible family.

Observation 3. If two effective chords of an admissible family for $P$ have a common vertex, then these two chords are collinear and their common vertex is an isolated point in $\mathrm{Bd} P$.

\section{Addition of Segments to Formal Boundary}

Any dissection of a rectilinear polygon into rectangles can be considered as a repeated addition of some closed line segments to the formal boundary of the polygon. In this section, we study some auxiliary results regarding this addition.

Let $[x, z] \subset P$ be a closed line segment (either horizontal or vertical) such that the intersection of $[x, z]$ with $\mathrm{Bd} P$ is a finite (possibly, empty) set. We can say that the polygon $P^{\prime}$ is obtained from $P$ by the addition of $[x, z]$ to $\mathrm{Bd} P$ provided:

(1) $\mathrm{Bd} P^{\prime}:=\mathrm{Bd} P \cup[x, z]$;

(2) Int $P^{\prime}:=$ Int $P \backslash[x, z]$;

(3) $V\left(P^{\prime}\right):=V(P) \cup\{x, z\} \cup($ Or $P \cap[x, z])$.

Observation 4. Segment $[x, z]$, added to $\mathrm{Bd} P$, is not, in general, an elementary segment of $\mathrm{Bd} P^{\prime}$; it is, however, a union of elementary segments of $\mathrm{Bd} P^{\prime}$.

We say that the addition of a segment $[x, z] \subset P$ to $\mathrm{Bd} P$ decreases by one the measure $m(z)$ of $P$ at a vertex $z \in V(P)$ if $m^{\prime}(z)=m(z)-1$, where $m^{\prime}(z)$ means the measure of $P^{\prime}$ at $z$.

Example. In Fig. 3, the addition of segment $[2,12]$ to $\mathrm{Bd} C$ decreases by one the measure of $C$ at each of the vertices 2,7 , and 12 , while the addition of segment $[11,12]$ to $\mathrm{Bd} C$ decreases the measure by one at only vertex 11 .

Lemma 3. Let $P^{\prime}$ be a polygon obtained from $P$ by the addition to $\operatorname{Bd} P$ of $a$ segment $[x, w] \subset P$ such that $w \in V(P)$. Then

$$
m(w)-1 \leq m^{\prime}(w) \leq m(w)
$$

where $m^{\prime}(w)$ denotes the measure of $P^{\prime}$ at $w$.

Proof. If $w$ is a point of local convexity of $P$, then trivially $m(w)=m^{\prime}(w)=0$. Assume that $w$ is a point of local nonconvexity of $P$ but not an isolated point in Bd $P$. Then $w$ is the apex of a concave inner angle of $P$. If $[X, w]$ does not intersect the interior of this angle, then $m(w)=m^{\prime}(w)=1$. Let $[x, w]$ divide this angle in two inner angles of $P^{\prime}$. If one of these new angles is concave, then $m(w)=\bar{m}^{t}(w)=1$. If both new angles are convex, then $m(w)-1=m^{\prime}(w)=0$.

Assume now that $w$ is an isolated point in $\operatorname{Bd} P$. Then $w$ is a vertex of a unique 
elementary segment $[u, w] \in S\left(P^{\prime}\right)$ (obviously, $[u, w]$ lies in $\left.[x, w]\right)$. In this situation $m(w)-1=m^{\prime}(w)=1$.

Lemma 4. Let $[v, w]$ be an effective chord of a rectilinear polygon $P$, and let $x_{1}, \ldots, x_{r}$ be all vertices of $P$ contained in $] v, w[$. The addition of $[v, w]$ to $\mathrm{Bd} P$ decreases by one the measure of $P$ at each of the points $v, w, x_{1}, \ldots, x_{r}$.

Proof. Each $x_{i}$ is a vertex of a unique elementary segment, say $\left[x_{i}, z_{i}\right]$, orthogonal to $[v, w]$ (see item 4 of Definition 7). In this case, $m\left(x_{i}\right)=1$ for all $i=1, \ldots, r$.

Denote by $P^{\prime}$ the polygon obtained from $P$ by the addition of $[v, w]$ to $\operatorname{Bd} P$. Relative to $P^{\prime}$, each vertex $x_{i}$ is the apex of three convex inner angles (two of them are of size $\pi / 2$ and one is of size $\pi$ ). Hence $x_{i}$ is a point of local convexity of $P^{\prime}$ and $m^{\prime}\left(x_{i}\right)=0$, where $m^{\prime}\left(x_{i}\right)$ denotes the measure of $P^{\prime}$ at $x_{i}$. Hence $m^{\prime}\left(x_{i}\right)=m\left(x_{i}\right)-1$.

If $v$ is an isolated point in $\operatorname{Bd} P$, then $v$ is a vertex of a unique elementary segment of the form $\left[v, x_{i}\right] \in S\left(P^{\prime}\right)$. In this situation, $m^{\prime}(v)=m(v)-1=1$. Assume that $v$ is not isolated in $\operatorname{Bd} P$. Then $v$ is a vertex of an elementary segment, say $[u, v] \in S(P)$, which is collinear to $[v, w]$ (see item 2 of Definition 7). In this situation, $v$ is a point of local convexity for $P^{\prime}$ and $m^{\prime}(v)=m(v)-1=0$. Similarly, $m^{\prime}(w)=m(w)-1$.

We need the following:

Definition 9. A line segment $[x, z]$ is called a simple chord of $P$ if the following conditions are fulfilled:

(1) $[x, z]$ is either horizontal or vertical;

(2) $x, z \in \mathrm{Bd} P$;

(3) the open interval $] x, z[$ is contained in Int $P$.

Observation 5. From Definitions 4 and 9 it follows that if a simple chord $[x, z]$ of $P$ is added to $\mathrm{Bd} P$, then $[x, z]$ becomes an elementary segment for the polygon $P^{\prime}$ such that

$$
\text { Bd } \left.P^{\prime}=\operatorname{Bd} P \cup[x, z], \quad \text { Int } P^{\prime}=\operatorname{Int} P \backslash\right] x, z\left[, \quad V\left(P^{\prime}\right)=V(P) \cup\{x, z\} .\right.
$$

Lemma 5. For any vertex $v$ of local nonconvexity of a rectilinear polygon $P$ there is a simple chord $[v, z]$ of $P$ whose addition to $\mathrm{Bd} P$ decreases by one the measure $m(v)$.

Proof. The vertex $v$ is either an isolated point in Bd $P$ or the apex of some inner concave angle of $P$. If $v$ is an isolated point in $\mathrm{Bd} P$, then we choose a horizontal segment $[v, z]$ such that $z \in \operatorname{Bd} P$ and $] v, z[\subset \operatorname{Int} P$. The chord $[v, z]$ is simple for $P$ and $m^{\prime}(v)=1$, where $m^{\prime}(v)$ denotes the measure at $v$ for the polygon $P^{\prime}$ obtained from $P$ by the addition of $[v, z]$ to $\mathrm{Bd} P$. In this case, $m^{\prime}(v)=m(v)-1=1$.

Assume now that $v$ is the apex of an inner concave angle of $P$ formed by 
elementary segments, say $[u, v],[v, w]$. Choose the chord $[z, v]$ such that:

(1) $[z, v]$ is collinear to $[v, w]$, and $v \in] z, x[$;

(2) $z \in \mathrm{Bd} P$ and $] z, v[\subset$ Int $P$.

The chord $[z, v]$ is simple for $P$ and $m^{\prime}(v)=m(v)-1=0$.

\section{Decomposition of Supplementary Ornament}

Assume that a rectilinear polygon $P$ is dissected into rectangles $R_{1}, \ldots, R_{q}$ (see Definition 2). Denote by $L_{1}, \ldots, L_{q}$ the boundary contours of $R_{1}, \ldots, R_{q}$, respectively, and put $L:=L_{1} \cup \cdots \cup L_{q}$.

Define $W$ to be set of vertices of $R_{1}, \ldots, R_{q}$. Then $V(P) \cup W$ divides the point-set union $L$ of all $R_{1}, \ldots, R_{q}$ sides into a family $\mathscr{S}$ of closed line segments. Therefore, we may consider $L$ as the formal boundary of the rectilinear polygon $P_{0}$ such that

$$
\text { Bd } P_{0}=L, \quad \text { Int } P_{0}=\text { Int } P \backslash L, \quad V\left(P_{0}\right)=V(P) \cup W, \quad S\left(P_{0}\right)=\mathscr{S} .
$$

Note that $P$ and $P_{0}$ have the same topological boundary and topological interior, and any connected component of Int $P_{0}$ is the interior of some rectangle $R_{i}$.

As mentioned in Section 5, polygon $P_{0}$ can be obtained from $P$ by the repeated addition of some closed line segments to $\mathrm{Bd} P$. In this section we investigate the inverse approach: to obtain $P$ from $P_{0}$ by the repeated deletion of some open line intervals. In this way, the "supplementary ornament" $Y:=L \backslash \mathrm{Bd} P$ will be studied.

Lemma 6. The set $Y=L \backslash \mathrm{Bd} P$ can be decomposed into pairwise disjoint open line intervals.

Proof. Denote by $\mathscr{L}$ the family of all maximal, with respect to inclusion, open intervals contained in $Y$. Since $Y$ is itself an open set, we have $Y=\bigcup\{l: l \in \mathscr{L}\}$. Generally speaking, $\mathscr{L}$ does not satisfy the condition of Lemma 6 because it may contain some elements with nonempty intersection. In order to obtain the desired decomposition, we execute repeatedly the following:

Segment splitting procedure. If open intervals $] x, y[] z,, v[$ in $\mathscr{L}$ have a common point $w$ (in this case, $] x, y[$ and $] z, v[$ are orthogonal to each other), then put $\mathscr{L}:=(\mathscr{L} \backslash] z, v[) \cup\{] z, w[] w, v]$,$\} . Since \mathscr{L}$ is finite, the required decomposition exists.

Example. Polygon $D$ (see Fig. 4) with the ornament Or $D=[1,4] \cup[2,3] \cup$ $[5,8] \cup\{6\}$ is dissected into four rectangles. The set $Y:=L \backslash \mathrm{Bd} D$ is decomposed into open intervals as follows: $Y=] 13,4[U] 4,5[\cup] 5,6[\cup] 6,7[$.

Let $\mathscr{L}=\left\{l_{1}, \ldots, l_{k}\right\}$ be a decomposition of $Y$ into pairwise disjoint open line intervals, and let $\left.l_{i}=\right] x_{i}, z_{i}\left[, i=1, \ldots, k\right.$. Denote by $P_{i}$ the rectilinear polygon obtained from $P_{0}$ by deleting $l_{1} \cup \cdots \cup l_{i}$ from $L, i=1, \ldots, k$.

Generally speaking, the polygon $P_{k}$ can be different from $P$ because it may contain some new vertices (as vertex 7 of $D_{0}$ for polygon $D$ in Fig. 4). At the same 


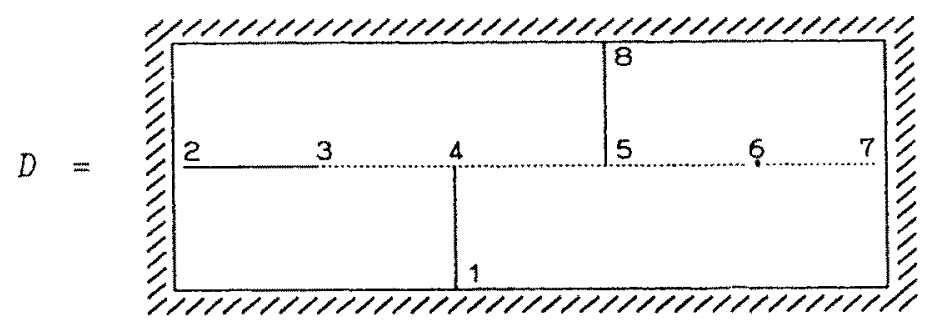

Fig. 4. "Supplementary ornament" of a rectangular dissection.

time $\mathrm{Bd} P_{k}=\mathrm{Bd} P$, and any vertex $w \in V\left(P_{k}\right) \backslash V(P)$ is a point of local convexity for $P$. Therefore $m\left(P_{k}\right)=m(P)$.

Since the set $L_{i}:=L \backslash\left(l_{1} \cup \cdots \cup l_{i}\right)$ may be nonclosed for $1 \leq \mathrm{i}<k$, the respective ornaments of $P_{1}, \ldots, P_{k-1}$ are not well defined (by definition, the ornament of a rectilinear polygon is a union of isolated points and closed segments). Therefore we cannot directly apply to $P_{i}$ the notion of measure of local nonconvexity. Nevertheless, we can avoid this obstacle for vertices of $P$ (but not for vertices of $\left.P_{i}\right)$ in the following way.

A set $L_{i}$ can be nonclosed if we delete, together with some interval $l_{p}, p \leq i$, an endpoint of an interval $l_{q}, q>i$. Since $l_{q} \cap \mathrm{Bd} P=\varnothing$, this deleted vertex cannot be a vertex of $\mathrm{Bd} P$. Hence for any vertex $w$ in $V(P)$, we can choose a small square $Q$ (having vertical and horizontal sides) with center $w$ such that the intersection of $Q$ with $L_{i}$ is closed. Now we define the measure $m_{i}(w)$ of $P_{i}$ at $w$ relative to that part of Bd $P_{i}$ which belongs to $Q$. Since the notion of measure is local, our approach is correct.

Definition 10. For any decomposition of the set $Y=L \backslash \mathrm{Bd} P$ into pairwise disjoint open intervals $\left.l_{i}=\right] x_{i}, z_{i}\left[, i=1, \ldots, k\right.$, define the numbers $\mu_{i}\left(l_{i}\right), i=1, \ldots, k$, as follows:

(1) $\mu_{i}\left(l_{i}\right)=0$ if $x_{i} \notin V(P)$ and $z_{i} \notin V(P)$;

(2) $\mu_{i}\left(l_{i}\right)=m_{i}\left(x_{i}\right)-m_{i-1}\left(x_{i}\right)$ if $x_{i} \in V(P)$ and $z_{i} \notin V(P)$;

(3) $\mu_{i}\left(l_{i}\right)=m_{i}\left(z_{i}\right)-m_{i-1}\left(z_{i}\right)$ if $x_{i}+V(P)$ and $z_{i} \in V(P)$;

(4) $\mu_{i}\left(l_{i}\right)=\left[m_{i}\left(x_{i}\right)-m_{i-1}\left(x_{i}\right)\right]+\left[m_{i}\left(z_{i}\right)-m_{i-1}\left(z_{i}\right)\right]$ if $x_{i} \in V(P)$ and $z_{i} \in V(P)$;

where $m_{j}(w)$ denotes the measure of $P_{j}$ at a vertex $w \in V(P)$.

From Lemma 3, it follows that

$$
0 \leq m_{i}\left(x_{i}\right)-m_{i-1}\left(x_{i}\right) \leq 1, \quad 0 \leq m_{i}\left(z_{i}\right)-m_{i-1}\left(z_{i}\right) \leq 1
$$

Hence, from Definition 10, we obtain

\section{Observation 6.}

(1) $0 \leq \mu_{i}\left(l_{i}\right) \leq 2$ for all $i=1, \ldots, k$;

(2) $\mu_{i}\left(l_{i}\right) \leq 1$ if at most one of $x_{i}, z_{i}$ is in $V(P)$;

(3) $\mu_{i}\left(l_{i}\right)=0$ if both vertices $x_{i}, z_{i}$ are not in $V(P)$. 
Observation 7. The values $\mu_{i}\left(l_{i}\right), i=1, \ldots, k$, depend on the order in which the intervals $l_{i}$ are considered (see example below).

The remainder of this section is devoted to the proof of:

Theorem 1. Let $\mathscr{L}=\left\{l_{1}, \ldots, l_{k}\right\}$ be a decomposition of the set $Y=L \backslash \mathrm{Bd} P$ into pairwise disjoint open line intervals. The intervals $l_{1}, \ldots, l_{k}$ can be renumbered so that $0 \leq \mu_{i}\left(l_{i}\right) \leq 1$ for at least $k-e$ of them, where $e$ denotes the effective number of $P$.

Before the proof of Theorem 1, we illustrate its statement with the help of the following example.

Example. Polygon $D$ in Fig. 4 has only one effective chord, namely, $[3,6]$. Hence $e(D)=1$. The set $Y=L \backslash \mathrm{Bd} D$ is decomposed into open line intervals ] $3,4[] 4,,5[] 5,,6[$, and $] 16,7[$. If we put

$$
\left.l_{1}=\right] 3,4\left[, \quad l_{2}=\right] 5,6\left[, \quad l_{3}=\right] 4,5\left[, \quad l_{4}=\right] 6,7[,
$$

then $\mu_{1}\left(l_{1}\right)=\mu_{2}\left(l_{2}\right)=2, \mu_{3}\left(l_{3}\right)=0$, and $\mu_{4}\left(l_{4}\right)=1$. If we renumber these intervals as

$$
\left.l_{1}=\right] 3,4\left[, \quad l_{2}=\right] 4,5\left[, \quad l_{3}=\right] 5,6\left[, \quad l_{4}=\right] 6,7[,
$$

then $\mu_{1}\left(l_{1}\right)=2$ and $\mu_{2}\left(l_{2}\right)=\mu_{3}\left(l_{3}\right)=\mu_{4}\left(l_{4}\right)=1$, as required in the statement of Theorem 1.

Proof of Theorem 1. An effective chord $[v, w]$ of $P$ is said to be compatible with $\mathscr{L}$ provided $[v, w] \backslash V(P)$ is a union of some intervals from $\mathscr{L}$.

Denote by $\mathscr{F}=\left\{t_{1}, \ldots, t_{f}\right\}$ an admissible family of effective chords of $P$ compatible with $\mathscr{L}$ and having maximum cardinality, and put $T:=t_{1} \cup \cdots \cup t_{f}$. Obviously, $f \leq e$.

Let $P_{T}$ be the region obtained from $P$ by the addition of $T$ to $\mathrm{Bd} P$; i.e., Bd $P_{T}=\operatorname{Bd} P \cup T$, Int $P_{T}=$ Int $P \backslash T$, and $V\left(P_{T}\right)=V(P)$.

Recall that $P_{i}$ denotes the rectilinear polygon obtained from $P_{0}$ by deleting $l_{1} \cup \cdots \cup l_{i}$ from $L, i=1, \ldots, k$.

First renumbering. Without loss of generality, we may suppose that the family $\left\{l_{1}, \ldots, l_{k}\right\}$ is renumbered such that, exactly the intervals $l_{1}, \ldots, l_{p}, p \leq k$ have no common vertex with $V(P)$, and all the other intervals $l_{p+1}, \ldots, l_{k}$ are as follows:

(1) each of the intervals $l_{p+1}, \ldots, l_{q}, q \leq k$, has exactly one vertex in $V(P)$;

(2) each of the intervals $l_{q+1}, \ldots, s_{s}, s \leq k$, is not contained in $T$ and has both vertices in $V(P)$;

(3) each of the intervals $l_{s+1}, \ldots, l_{k}$ is contained in $T$; i.e.,

$$
l_{s+1} \cup \cdots \cup l_{k}=T \backslash V(P) .
$$


Obviously, $\mathrm{Bd} P \subset \mathrm{Bd} P_{T} \subset \mathrm{Bd} P_{i}$ for all $i=0,1, \ldots, s$.

Due to Observation 6,

$$
\begin{aligned}
\mu_{i}\left(l_{i}\right)=0 & \text { for all } \quad \\
0 \leq \mu_{i}\left(l_{i}\right) \leq 1 & \text { for all } \quad i=p+1, \ldots, p,
\end{aligned}
$$

Second renumbering is organized for intervals $l_{q+1}, \ldots, l_{s}$ according to the following procedure:

1. Put $i=q+1$ and $k=0$.

2. If $\mu_{i}\left(l_{i}\right) \leq 1$, then put $k=0$ and go to 3 , elese go to 4 .

3. If $i<s$, then put $i=i+1$ and go to 2 , else exit.

4. If $k<s-i-1$, then put $k=k+1$, renumber $l_{i+1}, l_{i+2}, \ldots, l_{s}, l_{i}$ by the indices $i, i+1, \ldots, s-1, s$, respectively, and go to 2 , else exit.

As a result we obtain a renumbered sequence $l_{q+1}, \ldots, l_{s}$ such that:

(1) $0 \leq \mu_{i}\left(l_{i}\right) \leq 1, i=q+1, \ldots, r$, for some natural $r$ between $q+1$ and $s$;

(2) $\mu_{r+1}\left(l_{r+1}\right)=2$ for any renumbering of $l_{r+1}, \ldots, l_{s}$ by the indices $r+1, \ldots, s$.

We are going to prove that the family $l_{r+1}, \ldots, l_{s}$ is empty; i.e., the following assertion is valid.

Lemma 7. $r=s$.

Proof. Suppose, on the contrary, that $r<s$. Choose in the family $l_{r+1}, \ldots, l_{s}$ a maximal subfamily $\mathscr{M}$ such that all the intervals of $\mathscr{M}$ lie on a common line without segment gaps and no common vertex of two intervals from $\mathscr{M}$ is an isolated vertex in Bd $P_{T}$. Without loss of generality, consider this line to be horizontal. After an appropriate renumbering of $l_{r+1}, \ldots, l_{s}$, we can represent $\mathscr{M}$ as the union of intervals

$$
\left.l_{r+1}=\right] x_{r+1}, z_{r+1}\left[, \ldots, l_{j}=\right] x_{j}, z_{j}[, \quad r+1 \leq j \quad(\leq s),
$$

such that $z_{i}=x_{i+1}, i=r+1, \ldots, j-1$ (in the case $r+1<j$ ).

We investigate some properties of the segment $\left[x_{r+1}, z_{j}\right]$, which are listed in Claims 1-5 below.

Claim 1. No vertical elementary segment of Bd $P_{r}$ is incident with a point of $l_{r+1} \cup \cdots \cup l_{s}$.

Indeed, assume that some vertical elementary segment $m=[v, w]$ of $\mathrm{Bd} P_{0}$ has its vertex $v$ in $l_{r+1} \cup \cdots \cup l_{s}$. Since $Y \cap \operatorname{Bd} P=\varnothing, v$ is not a vertex of $P$. Hence $m$ is not in $\mathrm{Bd} P$; i.e., $m$ is contained in some interval $l_{i} \in \mathscr{L}$. Due to First Renumbering, we have $i \leq q$. Hence $m \cap \mathrm{Bd} P_{\mathrm{r}}=\varnothing$. 
Claim 2. Each of the vertices $x_{r+1}, z_{r+1}, \ldots, z_{j}$ is incident with at most one vertical elementary segment of $\mathrm{Bd} P_{r}$.

Suppose, on the contrary, that $x_{r+1}$ is incident with two vertical elementary segments of $\mathrm{Bd} P_{r}$ (points $z_{r+1}, \ldots, z_{j}$ are handled analogously). In this case, any interior angle of $P_{r+1}$ with the apex $x_{r+1}$ is of size $\pi$ or $\pi / 2$; i.e., $x_{r+1}$ is a point of local convexity for $P_{r+1}$. Hence $m_{r+1}\left(x_{r+1}\right)=0$. Similarly, $m_{r}\left(x_{r+1}\right)=0$. By Definition $10, \mu_{r+1}\left(l_{r+1}\right) \leq 1$, which is in contradiction to the condition $\mu_{r+1}\left(l_{r+1}\right)=2$, by the choice of $l_{r+1}$.

Claim 3 Each of the vertices $z_{r+1}, \ldots, z_{j-1}$ (in the case $r+1<j$ ) is incident with a unique vertical elementary segment of $\mathrm{Bd} P_{T}$.

By the choice of $\mathscr{M}$, each of $z_{r+1}, \ldots, z_{j-1}$ is not isolated in $\mathrm{Bd} P_{T}$. Hence, for each $z_{k}, k=r+1, \ldots, j-1$, there exists at least one vertical elementary segment of Bd $P_{T}$ whose end is $z_{k}$. Due to Claim 2, there exists at most one elementary segment of $\mathrm{Bd} P_{T}$ whose end is $z_{k}$. Since $\mathrm{Bd} P_{T} \subset \mathrm{Bd} P_{r}$, there exists exactly one elementary segment of $\mathrm{Bd} P_{r}$ whose end is $z_{k}$ and this segment belongs to $\mathrm{Bd} P_{T}$.

Claim 4. Each of $x_{r+1}, z_{j}$ is either an isolated point in $\mathrm{Bd} P_{T}$ or a vertex of a horizontal elementary segment of $\mathrm{Bd} P_{T}$.

In order to obtain a contradiction, suppose that $x_{r+1}$ is neither an isolated vertex in $\mathrm{Bd} P_{T}$ nor a vertex of a horizontal segment of $\mathrm{Bd} P_{T}$ (for $z_{j}$, the consideration is similar). Then $x_{r+1}$ is a vertex of a vertical elementary segment of $\mathrm{Bd} P_{T}$. Due to Claim 2, there is exactly one vertical elementary segment of Bd $P_{T}$ incident with $x_{r+1}$.

If $\mathrm{Bd} P_{r+1}$ were to contain no horizontal elementary segment incident with $x_{r+1}$, then the deletion of $l_{r+1}$ from Bd $P_{r}$ would keep the measure of $P_{r}$ at $x_{r+1}: m_{r}\left(x_{r+1}\right)=m_{r+1}\left(x_{r+1}\right)=1$. In this case, $\mu_{r+1}\left(l_{r+1}\right) \leq 1$, which is impossible by the choice of $l_{r+1}$. Hence Bd $P_{r+1}$ contains a horizontal elementary segment, say $\left[v, x_{r+1}\right]$.

By this assumption, $\left[v, x_{r+1}\right]$ is not in $\mathrm{Bd} P_{T}$. Hence $\left[v, x_{r+1}\right]$ is contained in a horizontal segment $l_{i}, i \geq r+2$. Due to the choice of $\mathscr{M}$, we have $i \geq s+1$. Therefore, by First Renumbering, $l_{i}$ belongs to a horizontal effective chord $t_{h} \in \mathscr{F}$. Since $l_{r+1}$ is not contained in $t_{h}, x_{r+1}$ is a vertex of $t_{h}$. In this case, $x_{r+1}$ is either an isolated point of $\mathrm{Bd} P$ or a vertex of a horizontal elementary segment of $\mathrm{Bd} P$ (see Definition 7). Since there is no horizontal elementary segment of $\mathrm{Bd} P$ incident with $x_{r+1}$, it is an isolated point in $\mathrm{Bd} P$. At the same time, we have shown above that $x_{r+1}$ is a vertex of a unique elementary segment of $\mathrm{Bd} P_{T}$. The last is possible only if this vertical segment belongs to $T$; i.e., $x_{r+1}$ is a vertex of some vertical effective chord $t_{v} \in \mathscr{F}$.

Thus we have found in $\mathscr{F}$ two orthogonal effective chords $t_{h}, t_{v}$ with a common vertex, which is impossible (see Definition 8). The obtained contradiction shows that each of $x_{r+1}, z_{j}$ is either an isolated point in $\mathrm{Bd} P_{T}$ or a vertex of a horizontal elementary segment of $\mathrm{Bd} P_{T}$. 
Claim 5. Neither $x_{p+1}$ nor $z_{j}$ is a vertex of a vertical effective chord from $\mathscr{F}$.

Consider the vertex $x_{r+1}$. By Claim 4, $x_{r+1}$ is either an isolated point in $\mathrm{Bd} P_{T}$ or a vertex of a horizontal elementary segment of $\mathrm{Bd} P_{T}$. If $x_{r+1}$ is isolated in $\mathrm{Bd} P_{T}$, then it cannot be a vertex of a vertical effective chord from $\mathscr{F}$. Let $x_{r+1}$ be a vertex of a horizontal elementary segment of $\mathrm{Bd} P_{T}$, say $\left[v, x_{r+1}\right]$. Assume, in order to obtain a contradiction, that $x_{r+1}$ is an end of a vertical chord $t_{g} \in \mathscr{F}$. Since no two orthogonal chords in $F$ have a common point (see Definition 8 ), the segment $\left[v, x_{r+1}\right]$ is not in $T$; i.e., $\left[v, x_{r+1}\right]$ is an elementary segment of $\mathrm{Bd} P$. Due to Definition 7 , there is a vertical elementary segment, say $\left[w, x_{r+1}\right]$, in $\mathrm{Bd} P$. Thus we obtained the existence of two vertical elementary segments of $\operatorname{Bd} P_{T}$ incident with $x_{r+1}$ (one of them is $\left[w, x_{r+1}\right]$ and another is contained in $t_{g}$ ), which is in contradiction to Claim 2.

Now we continue the proof of Lemma 7 . Claims $1-4$ mean that $\left[x_{r+1}, z_{j}\right]$ is an effective chord of $P$ compatible with the family $\mathscr{L}$. Due to the choice of $\mathscr{F}$, the chord $\left[x_{r+1}, z_{j}\right]$ cannot be included in $\mathscr{F}$. Hence there is at least one vertical effective chord in $\mathscr{F}$ having a vertex in $\left[x_{r+1}, z_{j}\right]$. By Claim 5 , no vertical effective chord in $\mathscr{F}$ in incident with $x_{r+1}$ or with $z_{j}$. Denote by $t_{i}^{\prime}=\left[z_{i}^{\prime}, w_{i}\right], i=1, \ldots, m$, all vertical chords of $\mathscr{F}$ having their vertices $z_{1}^{\prime}, \ldots, z_{m}^{\prime}$ in $] x_{r+1}, z_{j}[$ (see Fig. 5 for an example of $\left[x_{r+1}, z_{j}\right]$ in the case $r+1=3$ and $j=7$; here unbroken lines denote elementary segments of $\mathrm{Bd} P$, wavy lines denote vertical effective chords of $\mathscr{F}$, and dotted lines denote elementary segments of $\mathrm{Bd} P_{r+1}$ which do not belong to $\mathrm{Bd} P_{T} ; z_{4}$ and $z_{5}$ are isolated points of $\mathrm{Bd} P$ ).

Since each of $z_{1}^{\prime}, \ldots, z_{m}^{\prime}$ is a vertex of exactly one vertical elementary segment of $\mathrm{Bd} P_{T}$ (see Claim 3 ) and each chord $t_{i}^{\prime}$ contains no elementary segment of $\mathrm{Bd} P$, the vertices $z_{1}^{\prime}, \ldots, z_{m}^{\prime}$ are isolated points in $\mathrm{Bd} P$. In this situation, the chords

$$
\left[x_{r+1}, z_{1}^{\prime}\right],\left[z_{1}^{\prime}, z_{2}^{\prime}\right], \ldots,\left[z_{m}^{\prime}, z_{j}\right]
$$

are effective for $P$. Put

$$
\mathscr{F}^{\prime}=\left(\mathscr{F} \cup\left\{\left[x_{r+1}, z_{1}^{\prime}\right],\left[z_{1}^{\prime}, z_{2}^{\prime}\right], \ldots,\left[z_{m}^{\prime}, z_{j}\right]\right) \backslash\left\{t_{1}^{\prime}, \ldots, t_{m}^{\prime}\right\}\right.
$$

It is easily seen that $\mathscr{F}^{\prime}$ is an admissible family of effective chords of $P$ compatible with $\mathscr{L}$, and card $\mathscr{F}^{\prime}=$ card $\mathscr{F}+1$. The last is in contradiction to the choice of $\mathscr{F}$. The obtained contradiction shows that the assumption $r<s$ is false.

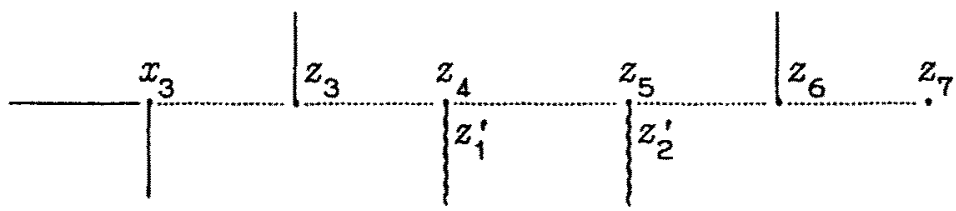

Fig. 5. 
In summary, we obtain that the Second Renumbering gives

$$
0 \leq \mu_{i}\left(l_{i}\right) \leq 1 \quad \text { for all } \quad i=q+1, \ldots, s
$$

Third Renumbering. It remains to renumber the intervals $l_{s+1}, \ldots, l_{k}$. Due to First Renumbering, these intervals are contained in the set $T=t_{1} \cup \cdots \cup t_{f}$. Without loss of generality, we may assume that the intervals $l_{s+1}, l_{s+2}, \ldots, l_{w}$, $w \leq k$, form $t_{1}$, i.e.,

$$
] x_{s+1}, z_{s+1}[\cup \cdots \cup] x_{w}, z_{w}\left[=t_{1} \backslash V(P),\right.
$$

such that $z_{i}=x_{i+1}, i=s+1, \ldots, w-1$ (in the case $s+1<w$ ).

From Definition 7, it follows that each of $z_{s+1}, \ldots, z_{w-1}$ is a vertex of some elementary segment $\left[z_{i}, u_{i}\right]$ of $\mathrm{Bd} P$ orthogonal to $t_{1}$, and each of $x_{s+1}, z_{w}$ is either an isolated point in $\mathrm{Bd} P_{T}$ or a vertex of a horizontal elementary segment of $\mathrm{Bd} P_{T}$. It is easily seen that $\mu_{s+1}\left(l_{s+1}\right)=2$, and $\mu_{i}\left(l_{i}\right)=1$ for all $i=s+2, \ldots, w$.

Similarly, we renumber repeatedly the intervals $l_{w+1}, \ldots, l_{k}$ such that for any chord $t_{i}, i=2, \ldots, f$, there exists exactly one interval $l_{i}$ contained in $t_{i}$ and having the respective number $\mu_{i_{r}}\left(l_{i_{r}}\right)=2$, while $\mu_{j}\left(l_{j}\right)=1$ for all other intervals $l_{j}$ in $t_{i}$.

Since $f \leq e$, the proof of Theorem 1 is complete.

\section{Auxiliary Results}

In this section we continue to study the "supplementary ornament" $Y=L \backslash \mathrm{Bd} P$. Let $\mathscr{L}=\left\{l_{1}, \ldots, l_{k}\right\}$ be a decomposition of $Y$ into pairwise disjoint open intervals.

Lemma 8. $m(P)=\mu_{1}\left(l_{1}\right)+\cdots+\mu_{k}\left(l_{k}\right)$.

Proof. If a vertex $w \in V(P)$ is not an end of $l_{i}$, then, by Definition $6, m_{i}(w)=$ $m_{i-1}(w)$. Therefore, for any $i=1, \ldots, k$,

$$
\mu_{i}\left(l_{i}\right)=\sum^{\prime}\left[m_{i}(w)-m_{i-1}(w)\right],
$$

where the sum $\sum^{\prime}$ is taken over the set of all vertices of $P$.

By Lemma $2, P_{0}$ has no points of local nonconvexity. Hence $m_{0}(w)=0$ for any vertex $w$ of $P$. In Section 6 , it was mentioned that $\mathrm{Bd} P_{k}=\mathrm{Bd} P$. Therefore, $m_{k}(w)=m(w)$ for all $w \in V(P)$. Thus

$$
\begin{aligned}
\sum_{i}^{k} \mu_{i}\left(l_{i}\right) & =\sum_{1}^{k}\left(\sum^{\prime}\left[m_{i}(w)-m_{i-1}(w)\right]\right) \\
& =\sum^{\prime} m_{k}(w)=\sum^{\prime} m(w)=m(P) .
\end{aligned}
$$


Lemma 9. The number $k$ of open intervals $l_{1}, \ldots, l_{k}$ decomposing the set $Y$ is at least $k \geq m-e$, where $m$ is the measure of local nonconvexity of $P$, and $e$ is the effective number of $P$.

Proof. By Theorem 1, the intervals $l_{1}, \ldots, l_{k}$ can be renumbered by the indices $1, \ldots, k$ such that $\mu_{i}\left(l_{i}\right) \leq 1$, for at least $k-e$ of them. Put $\mathscr{L}_{r}=$ $\left\{l_{i} \in \mathscr{L}: \mu_{i}\left(l_{i}\right)=r\right\}, r=0,1,2$. Since $0 \leq \mu_{i}\left(l_{i}\right) \leq 2$ for all $i=1, \ldots, k$, we have $\mathscr{L}=\mathscr{L}_{0} \cup \mathscr{L}_{1} \cup \mathscr{L}_{2}$ and

$$
\mu_{1}\left(l_{1}\right)+\cdots+\mu_{k}\left(l_{k}\right)=\left|\mathscr{L}_{1}\right|+2\left|\mathscr{L}_{2}\right|
$$

By the above, $\left|\mathscr{L}_{2}\right| \leq e$. Now from Lemma 8 , it follows that

$$
\begin{aligned}
k & =\left|\mathscr{L}_{0}\right|+\left|\mathscr{L}_{1}\right|+\left|\mathscr{L}_{2}\right| \geq\left(\left|\mathscr{L}_{1}\right|+2\left|\mathscr{L}_{2}\right|\right)-\left|\mathscr{L}_{2}\right| \\
& =\mu_{1}\left(l_{1}\right)+\cdots+\mu_{k}\left(l_{k}\right)-\left|\mathscr{L}_{2}\right| \geq m-e .
\end{aligned}
$$

The next lemma contains another estimate for the number $k$ of intervals $l_{1}, \ldots, l_{k}$.

Denote by $\alpha_{0}$ and $\alpha_{1}$, respectively, the numbers of all vertices and all elementary segments of a polygon $P$. The respective numbers for the polygon $P_{0}$ are denoted by $\beta_{0}$ and $\beta_{1}$ (see Section 6 for the definition of $P_{0}$ ).

Lemma 10. If the set $Y=L \backslash \mathrm{Bd} P$ is represented as a union of $k$ pairwise-disjoint open intervals $l_{1}, \ldots, l_{k}$, then

$$
k=\left(\beta_{1}-\alpha_{1}\right)-\left(\beta_{0}-\alpha_{0}\right) .
$$

Proof. Let us represent $\beta_{0}$ and $\beta_{1}$ as

$$
\beta_{0}=\gamma_{0}+\delta_{0}, \quad \beta_{1}=\gamma_{1}+\delta_{1},
$$

where $\gamma_{0}, \gamma_{1}$ denote, respectively, the number of $P_{0}$ vertices situated in $Y$ and the number of Bd $P_{0}$ elementary segments contained in the closure $\bar{Y}$, and let $\delta_{0}, \delta_{1}$ denote, respectively, the number of vertices of $P_{0}$ and the number of elementary segments of $\mathrm{Bd} P_{0}$ contained in $\mathrm{Bd} P$. Each interval $l_{i}$ can be decomposed as

$$
\left.l_{i}=\right] v_{1}, v_{2}\left[\cup\left\{v_{2}\right\} \cup \cdots \cup\left\{v_{r-1}\right\} \cup\right] v_{r-1}, v_{r}[, \quad r \geq 2
$$

where $\left[v_{i}, v_{i+1}\right], i=1, \ldots, r-1$, are elementary segments of bd $P_{0}$, and $v_{1}, \ldots, v_{r}$ are vertices of $P_{0}$; i.e., $l_{i}$ contains the interiors of $r-1$ elementary segments of Bd $P_{0}$ and $r-2$ vertices of $P_{0}$. Hence $\gamma_{1}-\gamma_{0}=k$.

If an elementary segment $[v, w]$ of $\mathrm{Bd} P$ contains in its interior some vertices $x_{1}, \ldots, x_{q}$ of $P_{0}$, then $[v, w]$ contains $q+1$ elementary segments of $\mathrm{Bd} P_{0}$. Hence $\delta_{0}-\alpha_{0}=\delta_{1}-\alpha_{1}$ and

$$
\left(\beta_{1}-\alpha_{1}\right)-\left(\beta_{0}-\alpha_{0}\right)=\left(\gamma_{1}-\gamma_{0}\right)+\left[\left(\delta_{1}-\alpha_{1}\right)-\left(\delta_{0}-\alpha_{0}\right)\right]=k
$$




\section{Generalized Euler's Relation}

The following lemma contains a generalized Euler's relation.

Lemma 11. For a rectilinear polygon $P$,

$$
\alpha_{0}-\alpha_{1}+c+h^{\prime}=c^{\prime}+h
$$

where

$\alpha_{0}$ is the number of vertices of $P$,

$\alpha_{1}$ is the number of elementary segments of $\mathrm{Bd} P$,

$c$ is the number of connected components of Int $P$,

$c^{\prime}$ is the number of connected components of $P$,

$h$ is the number of formal holes of $P$,

$h^{\prime}$ is the number of topological holes of $P$.

Proof. Consider the planar graph $G$, whose vertices and edges are precisely the vertices and the elementary segments of $P$, respectively. Hence $G$ has $\alpha_{0}$ vertices and $\alpha_{1}$ edges. Denote by $q$ the number of connected components of $E \backslash G$ (in other words, $G$ partitions $E$ into $q$ open connected domains). A well-known assertion states that

$$
q=\alpha_{1}-a_{0}+p+1
$$

where $p$ is the number of connected components of $G$. In our notations, $q=h^{\prime}+$ $c+1$, where 1 stands for Ext $P$.

We shall prove the equality $p=c^{\prime}+h$ by induction on $h$. The case $h=0$ is trivial: $p=c^{\prime}$. Suppose that the assertion " $p=c^{\prime}+h$ " is true for all rectilinear polygons with at most $h \leq l-1$ formal holes $(l \geq 1)$, and let $P$ be a rectilinear polygon with $l$ formal holes. Let $H$ be any formal hole of $P$. Then $H$ is separated from other formal holes or from Ext $P$ by a connected component $Q$ of Int $P$. It is possible to draw inside $Q$ an open simple polygonal rectilinear path $] x_{1}, \ldots, x_{m}[$ such that $x_{1}$ belongs to $H$ and $x_{m}$ belongs either to another formal hole or to Ext $P$. In any case, the addition of $\left[x_{1}, \ldots, x_{m}\right]$ to $\mathrm{Bd} P$ reduces by one both numbers $p$ and $l$. By inductive assumption, $p-1=c^{\prime}+l-1$ and $p=c^{\prime}+h$. According to all the data mentioned above, $\alpha_{0}-\alpha_{1}+c+h^{\prime}=c^{\prime}+h$.

Example. In Fig. $1, \alpha_{0}=32, \alpha_{1}=32, c=3, c^{\prime}=1, h=5$, and $h^{\prime}=3$.

\section{Minimum Number of Rectangles}

The following result was announced without proof in [10] (see also [11]).

Theorem 2. The minimum number of rectangles dissecting a rectilinear polygon $P$ 
is equal to

$$
m+c-h-e
$$

where

$m$ is the measure of $P$,

$c$ is the number of connected components of Int $P$,

$h$ is the number of formal holes of $P$,

$e$ is the effective number of $P$.

Proof. Let $r$ denote the minimum number of rectangles dissecting a rectilinear polygon $P$. First, we shall prove the inequality

$$
r \leq m+c-h-e .
$$

Suppose that $e>0$, and let $\left[x_{i}, z_{i}\right], i=1, \ldots, e$, be special chords of $P$ forming an admissible family of maximum cardinality. Denote by $P_{1}$ the polygon obtained from $P$ by the addition of $\left[x_{1}, z_{1}\right]$ to Bd $P$. Some vertices of $P$ can be situated in ]$x_{1}, z_{1}\left[\right.$. Denote them by $v_{1}, \ldots, v_{t}$, respectively. According to Definition 7 , any vertex $v_{i}$ is an end of some elementary segment from $\mathrm{Bd} P$ orthogonal to $\left[x_{1}, z_{1}\right]$. Hence the $\left[x_{1}, z_{1}\right]$ addition to $\mathrm{Bd} P$ leads to the following changes:

(1) the measure of $P$ at each of the points $x_{1}, v_{1}, \ldots, v_{t}, z_{1}$ decreases by one (see Lemma 4);

(2) the number $h=h(P)$ of formal holes of $P$ decreases by a number $p(\geq 0)$ and the number $c=c(P)$ of connected components of Int $P$ increases by a number $q(\geq 0)$ such that $p+q=t+1$

(3) the effective number $e(P)$ decreases by at most one.

Thus

$$
\begin{array}{ll}
m\left(P_{1}\right)=m-t-2, & c\left(P_{1}\right)=c+q, \\
h\left(P_{1}\right)=h-p, & e\left(P_{1}\right) \geq e-1,
\end{array}
$$

and

$$
m\left(P_{1}\right)+c\left(P_{1}\right)-h\left(P_{1}\right)-e\left(P_{1}\right) \leq m+c-h-e
$$

Obviously, the admissibility condition implies that chords $\left[x_{i}, z_{i}\right], i=2, \ldots, e$, remain effective for $P_{1}$.

After repeated additions of the chords $\left[x_{i}, z_{i}\right], i=2, \ldots, e$, to $\operatorname{Bd} P_{1}$, we obtain a rectilinear polygon $P_{e}$ such that

$$
\text { Bd } P_{e}=\operatorname{Bd} P \cup\left(\bigcup_{i=1}^{e}\left[x_{i}, z_{i}\right]\right), \quad V\left(P_{e}\right)=V(P) .
$$


As before,

$$
m\left(P_{e}\right)+c\left(P_{e}\right)-h\left(P_{e}\right)-e\left(P_{e}\right) \leq m+c-h-e .
$$

We claim that $e\left(P_{e}\right)=0$. Indeed, if there were at least one effective chord, $l$, of $P_{e}$, then, according to Definition 7 , their family $\left\{l,\left[x_{i}, z_{i}\right], i=1, \ldots, e\right\}$ would be an admissible family of cardinality $e+1$ for $P$, which is impossible. Hence

$$
m\left(P_{e}\right)+c\left(P_{e}\right)-h\left(P_{e}\right) \leq m+c-h-e .
$$

Assume that $m\left(P_{e}\right)>0$. Then some point $u$ of local nonconvexity of $P_{e}$ exists. By Lemma 5, there is a simple chord $[u, w]$ of $P_{e}$ whose addition to $\mathrm{Bd} P_{e}$ decreases by one the measure $m(u)$ of $P_{e}$. Denote by $P^{\prime}$ the polygon obtained from $P_{e}$ by the addition of $[u, w]$ to $\mathrm{Bd} P_{e}$. The addition of $[u, w]$ to $\mathrm{Bd} P_{e}$ decreases by one the measure of $P_{e}$ at $u$. Also, this addition either decreases by one the number $h\left(P_{\mathfrak{e}}\right)$ or increases by one the number $c\left(P_{\mathfrak{q}}\right)$. So

$$
m\left(P^{\prime}\right)+c\left(P^{\prime}\right)-h\left(P^{\prime}\right) \leq m+c-h-e .
$$

After repeated application of the above procedure of a simple chord addition, we obtain some rectilinear polygon $\tilde{P}$ without points of local nonconvexity; i.e., $m(\tilde{P})=0$. By Lemma $1, h(\tilde{P})=0$. Similarly to the above,

$$
c(\widetilde{P})=m(\widetilde{P})+c(\tilde{P})-h(\tilde{P}) \leq m+c-h-e .
$$

Due to Lemma 2, each connected component of Int $\tilde{P}$ is an open rectangle. The closures of these open rectangles dissect $\tilde{P}$ (and $P$ ) into $c(\tilde{P}$ ) rectangles. Therefore

$$
r \leq c(\tilde{P}) \leq m+c-h-e
$$

Now we prove the opposite inequality. Let $Q$ be a polygon obtained from $P$ by the addition of some line segments to $\mathrm{Bd} P$ such that $\mathrm{Bd} Q$ divides Int $Q$ into rectangles. Denote, respectively, by $\gamma_{0}$ and $\gamma_{1}$ the number of vertices and elementary segments of the polygon $Q$. By Lemma 11 ,

$$
\begin{aligned}
\alpha_{0}-\alpha_{1}+c+h^{\prime} & =c^{\prime}+h, \\
\gamma_{0}-\gamma_{1}+c(Q)+h^{\prime}(Q) & =c^{\prime}(Q)+h(Q),
\end{aligned}
$$

for polygons $P$ and $Q$, respectively. It is easily seen that

$$
h(Q)=0, \quad c^{\prime}(Q)=c^{\prime}, \quad h^{\prime}(Q)=h^{\prime} .
$$

Therefore

$$
c(Q)=c-h+\left[\left(\gamma_{1}-\alpha_{1}\right)-\left(\gamma_{0}-\alpha_{0}\right)\right]
$$


Lemmas 9 and 10 give

$$
\left(\gamma_{1}-\alpha_{1}\right)-\left(\gamma_{0}-\alpha_{0}\right)=(k) \geq m-e .
$$

Thus

$$
c(Q) \geq c-h+m-e .
$$

As $Q$ has no points of local nonconvexity, and connected component of Int $Q$ is an open rectangle (see Lemma 2). The closures of these open rectangles dissect $Q$ (and $P$ ) into $c(Q)$ rectangles. Hence any dissection of $P$ contains at least $m+c-h-e$ rectangles. In particular, $r \geq m+c-h-e$.

\section{Optimum Dissection Algorithm}

Theorem 2 not only gives a formula for a minimum number of dissecting rectangles, but also contains an informal description of a dissection algorithm which yields a minimum number of rectangles. Below, we describe this algorithm more precisely and show that its computational complexity is at most $O\left(n^{3 / 2} \log n\right)$.

Let $P$ be any rectilinear polygon in the plane $E$. We assume that the set $S(P)$ of all elementary segments of $P$ is given by two linear arrays forming, respectively, the topological boundary bd $P$ and the ornament Or $P$. Any vertex $v$ of $P$ is given by its Cartesian coordinates $(X, Y)$. Denote by $n$ the number of vertices of $P$.

\section{OPTIMUM DISSECTION ALGORITHM:}

1. Find the family $\mathscr{M}$ of all effective chords of $P$.

2. Select in $\mathscr{L}$ some admissible family $\mathscr{M}=\left\{s_{1}, \ldots, s_{e}\right\}$ of maximum cardinality.

3. Form the polygon $P_{m}$, adding $M:=\bigcup_{i=1}^{e} s_{i}$ to $\mathrm{Bd} P$.

4. Dissect $P_{M}$ into rectangles by drawing, in any order, inside $P_{M}$ some simple chords which remove the local nonconvexity of $P_{m}$ at each of its vertices.

We discuss each of Steps 1-4 separately.

1. The construction of the family $\mathscr{L}$ is organized for horizontal and vertical chords apart.

Procedure for finding effective horizontal chords:

(a) Form the family $\mathscr{H}$ of all horizontal chords $[x, z]$ such that $x, z \in V(P)$ and $] x, z[\subset \operatorname{Int} P$ (see [2]).

(b) Delete repeatedly from any chord $[x, z]$ for which two vertical elementary segments of the form $[x, v],[x, w]$ exist in $\mathrm{Bd} P$.

(c) Delete repeatedly from $\mathscr{H}$ each pair of chords $[x, v],[v, w]$ for which the vertex $v$ is not an isolated point in $\operatorname{Bd} P$ and include $[x, w]$ in $\mathscr{H}$. 
(d) Delete repeatedly from $\mathscr{H}$ any chord $[x, z]$ such that at least one of $x, z$ is not an isolated vertex of $\mathrm{Bd} P$ or a vertex of a horizontal elementary segment of $\mathrm{Bd} P$.

From Definition 7 , it follows that the repeated execution of (a)-(d) yields the family $\mathscr{H}=\left\{l_{1}, \ldots, l_{p}\right\}$ of all horizontal effective chords of $P$.

The family $\mathscr{V}=\left\{m_{1}, \ldots, m_{q}\right\}$ of all vertical effective chords of $P$ is constructed similarly.

In both cases a sweep-line technique is used. Hence the family $\mathscr{L}:=\mathscr{H} \cup \mathscr{V}$ can be found in $O(n \log n)$ time.

2. For the selection of some admissible family of maximum cardinality in $\mathscr{L}$, we use the appropriate $O\left(n^{3 / 2} \log n\right)$ algorithm from [3]. In [3], the family $\mathscr{L}$ is supposed to have the following property: no two collinear chords in $\mathscr{L}$ have a common point. However, in our case some two collinear chords in $\mathscr{L}$ may have a common point (see Observation 3). Hence for the implementation of step 2 in the dissection algorithm, we do the following:

(1) Transform (one-to-one) the family $\mathscr{L}$ of effective chords of $P$ into a family $\mathscr{L}^{\prime}$ of segments such that:

(i) a chord $[x, z] \in \mathscr{L}$ is horizontal (vertical) if and only if the corresponding segment $\left[x^{\prime}, z^{\prime}\right] \in \mathscr{L}^{\prime}$ is horizontal (vertical);

(ii) any two orthogonal chords $[x, z],[u, v] \in \mathscr{L}^{\prime}$ have a common point if and only if the corresponding segments $\left[x^{\prime}, z^{\prime}\right],\left[u^{\prime}, v^{\prime}\right] \in \mathscr{L}^{\prime}$ have a common point; and

(iii) no two collinear segments in $\mathscr{L}^{\prime}$ have a common point.

(2) Select in $\mathscr{L}^{\prime}$ an admissible family $\mathscr{M}^{\prime}=\left\{s_{1}^{\prime}, \ldots, s_{e}^{\prime}\right\}$ of maximum cardinality (using [3]),

(3) Obtain in $\mathscr{L}$ the corresponding family $\mathscr{M}=\left\{s_{1}, \ldots, s_{e}\right\}$ of special chords of $P$, using the inverse transformation $\mathscr{L}^{\prime} \rightarrow \mathscr{L}$.

Now we describe the $\mathscr{L} \rightarrow \mathscr{L}^{\prime}$ transformation. Let

$$
l_{i}=\left[x_{i}, v_{i}\right], \quad i=1, \ldots, p, \quad m_{j}=\left[u_{j}, w_{j}\right], j=1, \ldots, q
$$

be, respectively, all the horizontal and vertical chords in $\mathscr{L}$.

\section{Procedure of $\mathscr{L} \rightarrow \mathscr{L}^{\prime}$ transformation:}

(a) Find a real number $\varepsilon>0$ such that $\|x-y\|>2 \varepsilon$ for any two different points $x, y$ in the set

$$
\left\{x_{1}, \ldots, x_{p}, v_{1}, \ldots, v_{p}, u_{1}, \ldots, u_{q}, w_{1}, \ldots, w_{q}\right\}
$$

where $\|\cdot\|$ denotes the Manhattan metric in $E$.

(b) For any horizontal chord $l_{i}=\left[x_{i}, v_{i}\right] \in \mathscr{L}$ with the ends

$$
x_{i}=(X, Y), \quad v_{i}=(Z, Y), \quad X<Z,
$$


put $l_{i}^{\prime}=\left[x_{i}^{\prime}, v_{i}^{\prime}\right] \in \mathscr{L}^{\prime}$ with the endpoints

$$
x_{i}^{\prime}=\left(X-\varepsilon, Y+\delta_{i}\right), \quad v_{i}^{\prime}=\left(Z+\varepsilon, Y+\delta_{i}\right),
$$

where $\delta_{i}:=\varepsilon i /(p+q+), i=1, \ldots, p$.

(c) For any vertical chord $m_{j}=\left[u_{j}, w_{j}\right] \in \mathscr{L}$ with the ends

$$
u_{j}=(X, Y), \quad w_{j}=(X, Z), \quad Y<Z,
$$

put $m_{j}^{\prime}=\left[u_{j}^{\prime}, w_{j}^{\prime}\right] \in \mathscr{L}^{\prime}$ with the endpoints

$$
u_{j}^{\prime}=\left(X+\delta_{j}, Y-\varepsilon\right), \quad w_{j}^{\prime}=\left(X+\delta_{j}, Z+\varepsilon\right),
$$

where $\delta_{j}:=\varepsilon j /(p+q+1), j=1, \ldots, q$.

Example. For family $\mathscr{L}$ represented in Fig. 6(a), the corresponding family $\mathscr{L}^{\prime}$ is shown in Fig. 6(b).

Because of the $O(n \log n)$ time complexity of $\mathscr{L} \rightarrow \mathscr{L}^{\prime}$ and $\mathscr{L}^{\prime} \rightarrow \mathscr{L}$ transformations, we have the $O\left(n^{3 / 2} \log n\right)$ time complexity of step 2 in the dissection algorithm.

3. Step $P \rightarrow P_{M}$ has $O(n \log n)$ time complexity (see [2] for technique details).

4. The dissection of $P_{M}$ into rectangles can be organized in the following manner:

(a) For each point $x$ of the local nonconvexity of $P_{M}$, which is either an isolated point in $\mathrm{Bd} P_{M}$ or a vertex of some horizontal segment of $\mathrm{Bd} P_{H}$, draw inside $P_{H}$ a horizontal simple chord $[x, z](] x, z\left[\subset \operatorname{Int} P_{M}\right.$ and $\left.z \in \operatorname{Bd} P_{M}\right)$.

(b) Form the polygon $P_{T}$, adding to $\mathrm{Bd} P_{M}$ all the chords obtained in (a).

(c) For each vertex $x$ of the local nonconvexity of $P_{T}$, draw inside $P_{T}$ a vertical simple chord $[x, v](] x, v\left[\subset \operatorname{Int} P_{T}\right.$ and $\left.v \in \mathrm{Bd} P_{T}\right)$.

The computational complexity of step 4 is equal to $O(n \log n)$ [2]. According to all the data mentioned above, the dissection algorithm has at most $O\left(n^{3 / 2} \log n\right)$ time complexity.

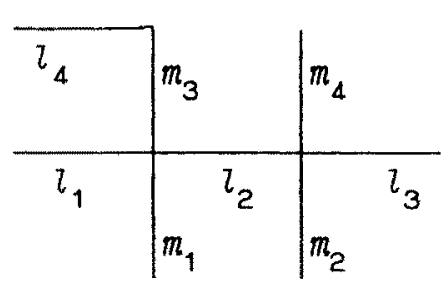

(a)

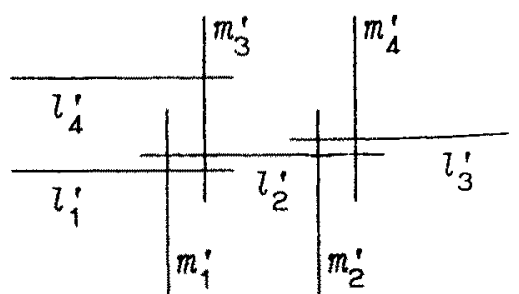

(b)

Fig. 6. An $\mathscr{L} \rightarrow \mathscr{L}^{\prime}$ transformation. 


\section{Acknowledgment}

The authors thank the referees for their many helpful comments on an earlier draft of the paper.

\section{References}

1. L. Ferrari, P. V. Sancar, and J. Sklansky, Minimal rectilinear partitions of digitized blocks, Comput. Vision Graphics Image Process. 28 (1984), 58-71.

2. A. N. Gorpinevich and V. Soltan, Simple algorithms of the partition of rectilinear regions into rectangles in VLSI engineering, Bull. Acad. Sci. Mold. SSR. Ser. Phis.-Tehn, and Math. Sci. No. 2 (1989), 19-25 (Russian).

3. H. Imai and T. Asano, Efficient algorithm for geometric graph search problems, SIAM J. Comput. 15 (1986), $478-494$.

4. N. M. Kornienko, G. V. Matveev, N. N. Metelsky and R. I. Tyshkevich, On tiling of polygons, Izv. Akad. Nauk. BSSR. Ser. Phis.-Math. Sci. No. 2 (1978), 25-29 (Russian).

5. A. Lingas, The power of non-rectilinear holes. Lecture Notes on Computer Science, Vol. 140. Springer-Verlag, Berlin, 1982, pp. 369-383.

6. W. Lipski, E. Lord, F. Luccio, C. Mugnai, and L. Pagli, On two-dimensional data organization II, Fund. Inform. 2 (1979), 245260.

7. W. Lipski, Finding a Manhattan path and related problems, Networks, 13 (1983), 399409.

8. W. Lipski, An $O(n \log n)$ Manhattan path algorithms, Inform. Process. Lett. $19(1984), 99-102$.

9. V. P. Rubtsov, Realization of VLSI topology by rectangles, Electronnaya Tehnica. Ser. 3 . No. 6 (1976), 54-61 (Russian).

10. V. Soltan and A. N. Gorpinevich, Minimum partition of a plane region into rectangles, In: Republican Seminar on Discrete Optimization, Kiev, 1985, pp. 107-108 (Russian).

11. V. Soltan and A. N. Gorpinevich, Some algorithms of the partition of a plane region into rectangles, In: Geometrie und Anwendungen. Vortrage der 7. Tagung der Fachsektion Geometrie. AdW der DDR, Berlin, 1988, pp. 111-112.

Received July 27, 1990, and in revised form November 10, 1991, and March 15, 1992. 